\title{
Stability for a Class of Differential Equations with Nonconstant Delay
}

\author{
Jin Liang, ${ }^{1}$ Tzon-Tzer Lu, ${ }^{2}$ and Yashan $\mathrm{Xu}^{3}$ \\ ${ }^{1}$ Department of Mathematics, Shanghai Jiao Tong University, Shanghai 200240, China \\ ${ }^{2}$ Department of Applied Mathematics, National Sun Yat-Sen University, Kaohsiung 80424, Taiwan \\ ${ }^{3}$ School of Mathematical Sciences, Fudan University, Shanghai 200433, China \\ Correspondence should be addressed to Jin Liang; jinliang@sjtu.edu.cn
}

Received 6 February 2013; Accepted 6 March 2013

Academic Editor: James H. Liu

Copyright (C) 2013 Jin Liang et al. This is an open access article distributed under the Creative Commons Attribution License, which permits unrestricted use, distribution, and reproduction in any medium, provided the original work is properly cited.

Stability is investigated for the following differential equations with nonconstant delay $x^{\prime}(t)=q(t) F(x(t))-p(t) f(x(t-\tau(t)))$, where $p:[0,+\infty) \rightarrow[0,+\infty), q:[0,+\infty) \rightarrow R, \tau:[0,+\infty) \rightarrow[0, r]$, and $F$ and $f: R \rightarrow R$ with $x f(x)>0$ for $x \neq 0$ and $|x| \leq a$ ( $a$ is a positive constant) are continuous functions. A criterion is given for the zero solution of this delay equation being uniformly stable and asymptotically stable.

\section{Introduction}

Delays are inherent in many physical and technological systems. In particular, pure delays are often used to ideally represent the effects of transmission, transportation, and inertia phenomena. Delay differential equations constitute basic mathematical models of real phenomena, for instance in biology, mechanics, and economics (cf., e.g., [1-17] and references therein). Stability analysis of delay differential equations is particularly relevant in control theory, where one cause of delay is the finite speed of communication. There have been a lot of results on the study of stability of delay differential equations. For example, we can see many earlier results on this issue from Burton's book [2]. Recently, in 2004, Butcher et al. [4] studied the stability properties of delay differential equations with time-periodic parameters. By employing a shifted Chebyshev polynomial approximation in each time interval with length equal to the delay and parametric excitation period, the system is reduced to a set of linear difference equations for the Chebyshev expansion coefficients of the state vector in the previous and current intervals. In 2005, Wahi and Chatterjee [16] used Galerkinprojection to reduce the infinite dimensional dynamics of a delay differential equation to one occurring on a finite number of modes. In 2009, Kalmár-Nagy [7] demonstrated that the method of steps for linear delay differential equation together with the inverse Laplace transform can be used to find a converging sequence of polynomial approximants to the transcendental function determining stability of the delay equation. Most recently, Berezansky and Braverman [3] gave some explicit conditions of asymptotic and exponential stability for the scalar nonautonomous linear delay differential equation with several delays and an arbitrary number of positive and negative coefficients.

This paper is concerned with the following differential equations with nonconstant delay:

$$
x^{\prime}(t)=q(t) F(x(t))-p(t) f(x(t-\tau(t))),
$$

where $a:[0,+\infty) \rightarrow[0,+\infty), q:[0,+\infty) \rightarrow R, \tau:$ $[0,+\infty) \rightarrow[0, r]$, and $F$ and $f: R \rightarrow R$ with

$$
x f(x)>0 \text { for } x \neq 0,|x| \leq a
$$

( $a$ is a positive constant) are continuous functions. We aim at giving general criterion for the zero solution of this delay equation being uniformly stable and asymptotically stable. 


\section{Main Result}

Denote by $C\left[t_{0}-r, t_{0}\right]$ the Banach space of continuous functions from $\left[t_{0}-r, t_{0}\right]$ to $R$ with the sup-norm

$$
\begin{gathered}
\|\varphi\|_{C\left[t_{0}-r, t_{0}\right]}=\max _{s \in\left[t_{0}-r, t_{0}\right]}\|\varphi(s)\|, \\
\quad \text { for every } \varphi \in C\left[t_{0}-r, t_{0}\right] .
\end{gathered}
$$

We consider (1) for $t \geq t_{0}$ with the initial conditions (for any $\left.t_{0} \geq 0\right)$

$$
x(t)=\varphi(t), \quad t_{0}-r \leq t \leq t_{0},
$$

where $\varphi \in C\left[t_{0}-r, t_{0}\right]$.

For an initial function $\varphi \in C\left[t_{0}-r, t_{0}\right]$, we denote by $x\left(t ; t_{0}, \varphi\right)$ the solution of (1) such that (4) holds.

Definition 1. The zero solution of (1) is said to be stable if for any $\varepsilon>0$ and $t_{0} \geq 0$, there exists $\delta\left(t_{0}, \varepsilon\right)>0$ such that if

$$
\|\varphi\|_{C\left[t_{0}-r, t_{0}\right]}<\delta
$$

then

$$
\left|x\left(t ; t_{0}, \varphi\right)\right|<\varepsilon \quad \forall t \geq t_{0} .
$$

The zero solution of (1) is uniformly stable if the above $\delta$ is independent of $t_{0}$.

Definition 2. The zero solution of (1) is said to be asymptotically stable if it is stable and if for any $t_{0} \geq 0$, there exists $\delta\left(t_{0}\right)>0$ such that if

$$
\|\varphi\|_{C[-r, 0]}<\delta
$$

then

$$
\left|x\left(t ; t_{0}, \varphi\right)\right| \longrightarrow 0, \quad \text { as } t \longrightarrow+\infty .
$$

Theorem 3. Assume that

(1) the zero solution to (1) is unique;

(2) if $q$ is nontrivial function and $F(\cdot)$ is nontrivial in any interval $[-b, b](b>0)$, then

$$
\begin{gathered}
\lim _{t \rightarrow+\infty} q(t)=0, \quad \lim _{t \rightarrow+\infty} \int_{t-\tau(t)}^{t}|q(s)| d s=0, \\
p(t) \geq \mu>0, \quad t \geq 0,
\end{gathered}
$$

for a constant $\mu$;

(3) $\lim _{t \rightarrow+\infty} \int_{t-\tau(t)}^{t} p(s) d s=A$;

(4) if $A \neq 0$, then

$$
|f(x)| \leq \frac{\lambda|x|}{2 A}, \quad \text { for } x \in R
$$

where $0<\lambda<1$.

Then the zero solution of (1) is uniformly stable.
Proof. For each $\varepsilon>0$, we set

$$
S(f, \varepsilon):=\sup \{|f(x)| ;|x| \leq \varepsilon\},
$$

and when $q$ is a nontrivial function and $F(\cdot)$ is nontrivial in any interval $[-b, b](b>0)$, we set

$$
S(F, \varepsilon):=\sup \{|F(x)| ;|x| \leq \varepsilon\},
$$

$$
I(\varepsilon):=\inf \{x f(y) ; x y>0,
$$

$$
\left.\frac{1-\lambda}{2} \varepsilon \leq|x| \leq \varepsilon, \frac{1-\lambda}{2} \varepsilon \leq|y| \leq \varepsilon\right\} .
$$

From (3) and (2), it follows that for every $\varepsilon>0$, there exists $t(\varepsilon)>0$ such that

$$
\begin{aligned}
& \int_{t-\tau(t)}^{t} p(s) d s \\
&< \begin{cases}\frac{1-\lambda}{4(S(f, \varepsilon)+1)} \varepsilon, & \text { if } A=0, \\
\frac{1-\lambda}{4(S(f, \varepsilon)+1)} \min \{A, 1\} \varepsilon+A, & \text { if } A \neq 0,\end{cases} \\
& \quad \forall t>t(\varepsilon),
\end{aligned}
$$

and when $q$ is a nontrivial function and $F(\cdot)$ is nontrivial in any interval $[-b, b](b>0)$, such that

$$
\begin{aligned}
& \int_{t-\tau(t)}^{t}|q(s)| d s<\frac{1-\lambda}{4(S(F, \varepsilon)+1)} \varepsilon, \quad \forall t>t(\varepsilon), \\
& |q(t)| \leq \mu \frac{I(\varepsilon)}{2(S(F, \varepsilon)+1)(\varepsilon+1)}, \quad \forall t>t(\varepsilon) .
\end{aligned}
$$

We claim that for any $\varepsilon>0$ and $t_{0} \geq t(\varepsilon)$, if

$$
\|\varphi\|_{C\left[t_{0}-r, t_{0}\right]}<\frac{1-\lambda}{2} \varepsilon
$$

then

$$
\left|x\left(t ; t_{0}, \varphi\right)\right|<\varepsilon \quad \forall t \geq t_{0},
$$

which means that the zero solution of (1) is eventually uniformly stable. Actually, if this is not true, then there exist

$$
\mathcal{E}_{0} \leq \min \{a, 1\}
$$

and a solution

$$
x(t):=x\left(t ; t_{0}, \varphi\right)
$$

to (1) with $\|\varphi\|_{C\left[t_{0}-r, t_{0}\right]}<((1-\lambda) / 2) \varepsilon$ and

$$
t_{0}>t\left(\varepsilon_{0}\right)
$$

such that there is a $\bar{t}>t_{0}$,

$$
|x(\bar{t})| \geq \varepsilon_{0} .
$$


Define

$$
\begin{gathered}
t_{2}:=\inf \left\{t \geq t_{0} ;|x(t)|=\varepsilon_{0}\right\}, \\
t_{1}:=\sup \left\{t_{0} \leq t<t_{2} ;|x(t)|=\frac{1-\lambda}{2} \varepsilon_{0}\right\}, \\
V(x)=x^{2}, \quad x \in R .
\end{gathered}
$$

Then, together with (21) and (22), we obtain

$$
\begin{gathered}
t\left(\varepsilon_{0}\right)<t_{1}<t_{2}, \\
V\left(x\left(t_{1}\right)\right)=\frac{(1-\lambda)^{2}}{4} \varepsilon_{0}^{2}, \quad V\left(x\left(t_{2}\right)\right)>\varepsilon_{0}^{2},
\end{gathered}
$$

and, for $t \in\left(t_{1}, t_{2}\right)$,

$$
\frac{(1-\lambda)^{2}}{4} \varepsilon_{0}^{2}<V(x(t))<\varepsilon_{0}^{2}
$$

and for arbitrary $\eta>0$, there exists $\xi \in\left[t_{2}-\eta, t_{2}\right]$ such that

$$
V^{\prime}(x(\xi))>0 .
$$

Therefore,

$$
V^{\prime}\left(x\left(t_{2}\right)\right) \geq 0
$$

This implies that

$$
t_{1} \geq t_{2}-\tau\left(t_{2}\right)
$$

In fact, if

$$
t_{1}<t_{2}-\tau\left(t_{2}\right)
$$

then by (23)-(25), we have

$$
\begin{gathered}
\frac{1-\lambda}{2} \varepsilon_{0} \leq\left|x\left(t_{2}-\tau\left(t_{2}\right)\right)\right| \leq \varepsilon_{0}, \\
t_{2}-\tau\left(t_{2}\right)>t\left(\varepsilon_{0}\right) .
\end{gathered}
$$

It is not hard to see that we can choose $t_{1}$ and $t_{2}$ above to make $x(t)$ have constant sign in $\left[t_{1}, t_{2}\right]$.

Case I. When $q(t) \equiv 0$ or

$$
F(x) \equiv 0 \quad \text { for }|x| \leq b,
$$

where $b$ is a positive real number.

In this case, if $q(t) \equiv 0$, then

$$
V^{\prime}\left(x\left(t_{2}\right)\right)=-2 p\left(t_{2}\right) x\left(t_{2}\right) f\left(x\left(t_{2}-\tau\left(t_{2}\right)\right)\right)<0,
$$

which contradicts with (28). Moreover, if

$$
F(x) \equiv 0 \text { for }|x| \leq b,
$$

for a positive real number $b$, then it is clear that we can require $\varepsilon_{0}<b$. Hence,

$$
V^{\prime}\left(x\left(t_{2}\right)\right)=-2 p\left(t_{2}\right) x\left(t_{2}\right) f\left(x\left(t_{2}-\tau\left(t_{2}\right)\right)\right)<0,
$$

which contradicts with (28) too.
Consequently, in this case we have the following observation.

Case I-1. If $A=0$, then we deduce by (23), (24), (1), and (11) that

$$
\begin{aligned}
\frac{\varepsilon_{0}}{2}+\frac{\lambda}{2} \varepsilon_{0} & =\left|x\left(t_{2}\right)\right|-\left|x\left(t_{1}\right)\right| \\
& \leq\left|x\left(t_{2}\right)-x\left(t_{1}\right)\right| \\
& \leq \int_{t_{1}}^{t_{2}} p(s)|f(x(s-\tau(s)))| d s \\
& +\int_{t_{1}}^{t_{2}}|q(s)||F(x(s))| d s \\
& \leq S\left(f, \varepsilon_{0}\right) \int_{t_{1}}^{t_{2}} p(s) d s \\
& \leq S\left(f, \varepsilon_{0}\right) \int_{t_{2}-\tau\left(t_{2}\right)}^{t_{2}} p(s) d s \\
& <\frac{\varepsilon_{0}}{2} .
\end{aligned}
$$

This is clearly impossible.

Case $I-2$. If $A \neq 0$, then we deduce by (23), (24), (1), (11), and (14) that

$$
\begin{aligned}
\frac{\varepsilon_{0}}{2}+\frac{\lambda}{2} \varepsilon_{0}= & \left|x\left(t_{2}\right)\right|-\left|x\left(t_{1}\right)\right| \\
\leq & \left|x\left(t_{2}\right)-x\left(t_{1}\right)\right| \\
\leq & \int_{t_{1}}^{t_{2}} p(s)|f(x(s-\tau(s)))| d s \\
& +\int_{t_{1}}^{t_{2}}|q(s)||F(x(s))| d s \\
\leq & \frac{\lambda \varepsilon_{0}}{2 A} \int_{t_{1}}^{t_{2}} p(s) d s \\
\leq & \frac{\lambda \varepsilon_{0}}{2 A} \int_{t_{2}-\tau\left(t_{2}\right)}^{t_{2}} p(s) d s \\
\leq & \frac{\lambda \varepsilon_{0}}{2 A}\left(\frac{1-\lambda}{4\left(S\left(f, \varepsilon_{0}\right)+1\right)} \min \{A, 1\} \varepsilon_{0}+A\right) \\
< & \frac{1-\lambda}{4} \varepsilon+\frac{\lambda}{2} \varepsilon \\
< & \frac{\varepsilon}{2} .
\end{aligned}
$$

This is clearly impossible too.

Therefore, in this case, the zero solution of (1) is eventually uniformly stable. This, together with assumption (1), implies that the zero solution of (1) is uniformly stable.

Case II. $q$ is a nontrivial function and $F(\cdot)$ is nontrivial in any interval $[-b, b](b>0)$. 
In this case, by virtue of (1), and assumption (2), (12), (13), and (16), we get

$$
\begin{aligned}
V^{\prime}\left(x\left(t_{2}\right)\right)= & -2 p\left(t_{2}\right) x\left(t_{2}\right) f\left(x\left(t_{2}-\tau\left(t_{2}\right)\right)\right) \\
& +2 x\left(t_{2}\right) q\left(t_{2}\right) F\left(x\left(t_{2}\right)\right) \\
\leq & -2 \mu I\left(\varepsilon_{0}\right)+2 \varepsilon_{0} \mu \frac{I\left(\varepsilon_{0}\right)}{2\left(S\left(F, \varepsilon_{0}\right)+1\right)\left(\varepsilon_{0}+1\right)} S(F, \varepsilon) \\
\leq & -\mu I(\varepsilon) \\
< & 0,
\end{aligned}
$$

which contradicts with (28). tion,

Consequently, in this case we have the following observa-

Case II-1. If $A=0$, then we deduce by (23), (24), (1), (11), (12), (14), and (15) that

$$
\begin{aligned}
\frac{\varepsilon_{0}}{2}+\frac{\lambda}{2} \varepsilon_{0}= & \left|x\left(t_{2}\right)\right|-\left|x\left(t_{1}\right)\right| \\
\leq & \left|x\left(t_{2}\right)-x\left(t_{1}\right)\right| \\
\leq & \int_{t_{1}}^{t_{2}} p(s)|f(x(s-\tau(s)))| d s \\
& +\int_{t_{1}}^{t_{2}}|q(s)||F(x(s))| d s \\
\leq & S(f, \varepsilon) \int_{t_{1}}^{t_{2}} p(s) d s \\
& +S(F, \varepsilon) \int_{t_{1}}^{t_{2}}|q(s)| d s \\
\leq & S(f, \varepsilon) \int_{t_{2}-\tau\left(t_{2}\right)}^{t_{2}} p(s) d s \\
& +S(F, \varepsilon) \int_{t_{2}-\tau\left(t_{2}\right)}^{t_{2}}|q(s)| d s \\
< & \frac{1-\lambda}{4} \varepsilon+\frac{1-\lambda}{4} \varepsilon \\
&
\end{aligned}
$$

This is a contradiction.

Case II-2. If $A \neq 0$, then we deduce by (23), (24), (1), (11), (12), (14), and (15) that

$$
\begin{aligned}
\frac{\varepsilon}{2}+\frac{\lambda}{2} \varepsilon & =\left|x\left(t_{2}\right)\right|-\left|x\left(t_{1}\right)\right| \\
& \leq\left|x\left(t_{2}\right)-x\left(t_{1}\right)\right|
\end{aligned}
$$

$$
\begin{aligned}
\leq & \int_{t_{1}}^{t_{2}} p(s)|f(x(s-\tau(s)))| d s \\
& +\int_{t_{1}}^{t_{2}}|q(s)||F(x(s))| d s \\
\leq & \frac{\lambda \varepsilon_{0}}{2 A} \int_{t_{1}}^{t_{2}} p(s) d s+S(F, \varepsilon) \int_{t_{1}}^{t_{2}}|q(s)| d s \\
\leq & \frac{\lambda \varepsilon_{0}}{2 A} \int_{t_{2}-\tau\left(t_{2}\right)}^{t_{2}} p(s) d s+S(F, \varepsilon) \int_{t_{2}-\tau\left(t_{2}\right)}^{t_{2}}|q(s)| d s \\
\leq & \frac{\lambda \varepsilon_{0}}{2 A}\left(\frac{1-\lambda}{4\left(S\left(f, \varepsilon_{0}\right)+1\right)} \min \{A, 1\} \varepsilon_{0}+A\right) \\
& +\frac{1-\lambda}{4} \varepsilon \\
< & \frac{1-\lambda}{4} \varepsilon+\frac{\lambda}{2} \varepsilon+\frac{1-\lambda}{4} \varepsilon \\
= & \frac{\varepsilon}{2} .
\end{aligned}
$$

This is a contradiction too.

Therefore, in this case, the zero solution of (1) is eventually uniformly stable. This, together with assumption (1), implies that the zero solution of (1) is uniformly stable.

Theorem 4. Assume that

(1) the zero solution to (1) is unique;

(2) if $q(t) \equiv 0$ or

$$
F(x) \equiv 0 \text { for }|x| \leq b,
$$

for a positive real number $b$, then

$$
\int_{0}^{+\infty} p(s) d s=+\infty
$$

(3) if $q$ is nontrivial function and $F(\cdot)$ is nontrivial in any interval $[-b, b](b>0)$, then

$$
\begin{gathered}
\lim _{t \rightarrow+\infty} q(t)=0, \quad \lim _{t \rightarrow+\infty} \int_{t-\tau(t)}^{t}|q(s)| d s=0, \\
\int_{0}^{+\infty} q(s) d s<+\infty, \\
p(t) \geq \mu>0, \quad t \geq 0,
\end{gathered}
$$

for a constant $\mu$;

(4) $\lim _{t \rightarrow+\infty} \int_{t-\tau(t)}^{t} p(s) d s=A$;

(5) if $A \neq 0$, then

$$
|f(x)| \leq \frac{\lambda|x|}{2 A}, \quad \text { for } x \in R,
$$

where $0<\lambda<1$. Then the zero solution of (1) is asymptotically stable. 
Proof. It follows from Theorem 3 that the zero solution of (1) is uniformly stable; that is, for arbitrarily given $\varepsilon>0$ and $t_{0} \geq 0$, there exists $\delta=\delta(\varepsilon)>0$ such that if

$$
\|\varphi\|_{C\left[t_{0}-r, t_{0}\right]}<\delta
$$

then

$$
\left|x\left(t ; t_{0}, \varphi\right)\right|<\varepsilon \quad \forall t \geq t_{0}
$$

Next, we will prove that

$$
\left|x\left(t ; t_{0}, \varphi\right)\right| \longrightarrow 0, \quad \text { as } t \longrightarrow+\infty .
$$

First, we show that

$$
\liminf _{t \rightarrow+\infty}\left|x\left(t ; t_{0}, \varphi\right)\right|=0 .
$$

Suppose that this is not true. Then

$$
\liminf _{t \rightarrow+\infty}\left|x\left(t ; t_{0}, \varphi\right)\right|>0 .
$$

Hence, for the arbitrarily given

$$
0<\varepsilon<\min \{a, b\},
$$

there exist $0<\varepsilon_{0}<\varepsilon$ and $T>t_{0}$ such that

$$
x\left(t ; t_{0}, \varphi\right)>\varepsilon_{0} \quad \forall t \geq T,
$$

or

$$
x\left(t ; t_{0}, \varphi\right)<-\varepsilon_{0} \quad \forall t \geq T .
$$

Let us now consider

$$
x\left(t ; t_{0}, \varphi\right)>\varepsilon_{0} \quad \forall t \geq T .
$$

Case I. When $q(t) \equiv 0$ or

$$
F(x) \equiv 0 \text { for }|x| \leq b,
$$

for a positive real number $b$, we obtain by assumption (2), (46), (50), and (53)

$$
\begin{aligned}
x(t)= & x(T+r)-\int_{T+r}^{t} p(s) f(x(s-\tau(s))) d s \\
& +\int_{T+r}^{t} q(s) F(x(s)) d s \\
\leq & x(T+r)-\inf \left\{f(x) ; x \in\left[\varepsilon_{0}, \varepsilon\right]\right\} \int_{T+r}^{t} p(s) d s .
\end{aligned}
$$

This implies that

$$
x(t) \longrightarrow-\infty \text { as } t \longrightarrow+\infty,
$$

which contradicts with (53).
Case II. When $q$ is a nontrivial function and $F(\cdot)$ is nontrivial in any interval $[-b, b](b>0)$, we obtain by assumptions (3), (46), (50), and (53)

$$
\begin{aligned}
x(t)= & x(T+r)-\int_{T+r}^{t} p(s) f(x(s-\tau(s))) d s \\
& +\int_{T+r}^{t} q(s) F(x(s)) d s \\
\leq & x(T+r)-\mu \inf \left\{f(x) ; x \in\left[\varepsilon_{0}, \varepsilon\right]\right\}(t-T-r) \\
& +\sup \left\{|F(x)| ; x \in\left(\varepsilon_{0}, \varepsilon\right)\right\} \int_{T+r}^{t}|q(s)| d s .
\end{aligned}
$$

This, together with assumption (2), implies that

$$
x(t) \longrightarrow-\infty \text { as } t \longrightarrow+\infty \text {, }
$$

which contradicts with (53).

Moreover, in a similar way, we can prove that

$$
x\left(t ; t_{0}, \varphi\right)<-\varepsilon \quad \forall t \geq T
$$

is impossible.

Therefore, (48) is true.

Based on (48), we will show that

$$
\limsup _{t \rightarrow+\infty}\left|x\left(t ; t_{0}, \varphi\right)\right|=0 .
$$

Actually, if this is not true, that is,

$$
\limsup _{t \rightarrow+\infty}\left|x\left(t ; t_{0}, \varphi\right)\right|>0,
$$

then by (48) we see that there are $\varepsilon_{0}$ with

$$
0<\varepsilon_{0}<\min \{a, b, 1\},
$$

and two sequences $\left\{\theta_{n}\right\}$ and $\left\{t_{n}\right\}$ such that

$$
\theta_{n}<t_{n}, \quad n=1,2, \ldots
$$

$$
\begin{gathered}
\theta_{n} \longrightarrow+\infty \quad t_{n} \longrightarrow+\infty \quad \text { as } n \longrightarrow+\infty, \\
V\left(x\left(\theta_{n}\right)\right)=\frac{(1-\lambda)^{2}}{4} \varepsilon_{0}^{2}, \quad V\left(x\left(t_{n}\right)\right)>\varepsilon_{0}^{2}, \\
V^{\prime}\left(x\left(t_{n}\right)\right)>0,
\end{gathered}
$$

and for $t \in\left(\theta_{n}, t_{n}\right)$

$$
\frac{(1-\lambda)^{2}}{4} \varepsilon_{0}^{2}<V(x(t))<\varepsilon_{0}^{2} .
$$

By the same reason as that in the proof of Theorem 3, we know that

$$
t_{n}-\tau\left(t_{n}\right) \leq \theta_{n} \leq t_{n} .
$$

Define $S(f, \varepsilon), S(F, \varepsilon), I(\varepsilon)$, and $t(\varepsilon)$ as those in the proof of Theorem 3. Then when $n$ is large enough, we have

$$
t_{n}>t(\varepsilon) \text {. }
$$


Case I. When $q(t) \equiv 0$ or

$$
F(x) \equiv 0 \text { for }|x| \leq b,
$$

where $b$ is a positive real number.

Case I-1. If $A=0$, then we deduce that

$$
\begin{aligned}
\frac{\varepsilon_{0}}{2}+\frac{\lambda}{2} \varepsilon_{0} & =\left|x\left(t_{n}\right)\right|-\left|x\left(\theta_{n}\right)\right| \\
\leq & \left|x\left(t_{n}\right)-x\left(\theta_{n}\right)\right| \\
\leq & \int_{\theta_{n}}^{t_{n}} p(s)|f(x(s-\tau(s)))| d s \\
& +\int_{\theta_{n}}^{t_{n}}|q(s)||F(x(s))| d s \\
\leq & S\left(f, \varepsilon_{0}\right) \int_{\theta_{n}}^{t_{n}} p(s) d s \\
\leq & S\left(f, \varepsilon_{0}\right) \int_{t_{n}-\tau\left(t_{n}\right)}^{t_{n}} p(s) d s \\
& <\frac{\varepsilon_{0}}{2} .
\end{aligned}
$$

This is impossible.

Case $I-2$. If $A \neq 0$, then we obtain

$$
\begin{aligned}
\frac{\varepsilon_{0}}{2}+\frac{\lambda}{2} \varepsilon_{0}= & \left|x\left(t_{n}\right)\right|-\left|x\left(\theta_{n}\right)\right| \\
\leq & \left|x\left(t_{n}\right)-x\left(\theta_{n}\right)\right| \\
\leq & \int_{\theta_{n}}^{t_{n}} p(s)|f(x(s-\tau(s)))| d s \\
& +\int_{\theta_{n}}^{t_{n}}|q(s)||F(x(s))| d s \\
\leq & \frac{\lambda \varepsilon_{0}}{2 A} \int_{\theta_{n}}^{t_{n}} p(s) d s \\
\leq & \frac{\lambda \varepsilon_{0}}{2 A} \int_{t_{n}-\tau\left(t_{n}\right)}^{t_{n}} p(s) d s \\
\leq & \frac{\lambda \varepsilon_{0}}{2 A}\left(\frac{1-\lambda}{4\left(S\left(f, \varepsilon_{0}\right)+1\right)} \min \{A, 1\} \varepsilon_{0}+A\right) \\
& <\frac{1-\lambda}{4} \varepsilon+\frac{\lambda}{2} \varepsilon \\
& <\frac{\varepsilon}{2} .
\end{aligned}
$$

This is clearly impossible too.
Consequently, (60) is true in this case.

Case II. When $q$ is nontrivial function and $F(\cdot)$ is nontrivial in any interval $[-b, b](b>0)$.

Case II-1. If $A=0$, then we deduce that

$$
\begin{aligned}
\frac{\varepsilon_{0}}{2}+\frac{\lambda}{2} \varepsilon_{0}= & \left|x\left(t_{n}\right)\right|-\left|x\left(\theta_{n}\right)\right| \\
\leq & \left|x\left(t_{n}\right)-x\left(\theta_{n}\right)\right| \\
\leq & \int_{\theta_{n}}^{t_{n}} p(s)|f(x(s-\tau(s)))| d s \\
& +\int_{\theta_{n}}^{t_{n}}|q(s)||F(x(s))| d s \\
\leq & S(f, \varepsilon) \int_{\theta_{n}}^{t_{n}} p(s) d s \\
& +S(F, \varepsilon) \int_{\theta_{n}}^{t_{n}}|q(s)| d s \\
\leq & S(f, \varepsilon) \int_{t_{n}-\tau\left(t_{n}\right)}^{t_{n}} p(s) d s \\
& +S(F, \varepsilon) \int_{t_{n}-\tau\left(t_{n}\right)}^{t_{n}}|q(s)| d s \\
< & \frac{1-\lambda}{4} \varepsilon+\frac{1-\lambda}{4} \varepsilon \\
& \varepsilon
\end{aligned}
$$

This is a contradiction.

Case $I I-2$. If $A \neq 0$, then we obtain

$$
\begin{aligned}
\frac{\varepsilon_{0}}{2}+\frac{\lambda}{2} \varepsilon_{0}= & \left|x\left(t_{n}\right)\right|-\left|x\left(\theta_{n}\right)\right| \\
\leq & \left|x\left(t_{n}\right)-x\left(\theta_{n}\right)\right| \\
\leq & \int_{\theta_{n}}^{t_{n}} p(s)|f(x(s-\tau(s)))| d s \\
& +\int_{\theta_{n}}^{t_{n}}|q(s)||F(x(s))| d s \\
\leq & \frac{\lambda \varepsilon_{0}}{2 A} \int_{\theta_{n}}^{t_{n}} p(s) d s+S(F, \varepsilon) \int_{\theta_{n}}^{t_{n}}|q(s)| d s \\
\leq & \frac{\lambda \varepsilon_{0}}{2 A} \int_{t_{n}-\tau\left(t_{n}\right)}^{t_{n}} p(s) d s \\
& +S(F, \varepsilon) \int_{t_{n}-\tau\left(t_{n}\right)}^{t_{n}}|q(s)| d s \\
\leq & \frac{\lambda \varepsilon_{0}}{2 A}\left(\frac{1-\lambda}{4\left(S\left(f, \varepsilon_{0}\right)+1\right)} \min \{A, 1\} \varepsilon_{0}+A\right)
\end{aligned}
$$




$$
\begin{aligned}
& +\frac{1-\lambda}{4} \varepsilon \\
< & \frac{1-\lambda}{4} \varepsilon+\frac{\lambda}{2} \varepsilon+\frac{1-\lambda}{4} \varepsilon \\
= & \frac{\varepsilon}{2} .
\end{aligned}
$$

This is a contradiction too.

Therefore, (60) is true in this case. So, (60) holds truly. This means that the zero solution of (4) is asymptotically stable.

Remark 5. Our results are new comparing with the results in $[2,3]$ since $\tau(t)$ could go to 0 or a big number as $t \rightarrow+\infty$ and in this case $p(t)$ also could be very large in our theorems. Moreover, for the case of $A=0$, the condition on $f$ in our results is very weak.

\section{Acknowledgment}

This work was supported by the NSF of China (11171210).

\section{References}

[1] R. P. Agarwal, C. Cuevas, and M. V. S. Frasson, "Semilinear functional difference equations with infinite delay," Mathematical and Computer Modelling, vol. 55, no. 3-4, pp. 1083-1105, 2012.

[2] T. A. Burton, Stability and Periodic Solutions of Ordinary and Functional-Differential Equations, Mathematics in Science and Engineering, Academic Press, Orlando, Fla, USA, 1985.

[3] L. Berezansky and E. Braverman, "New stability conditions for linear differential equations with several delays," Abstract and Applied Analysis, vol. 2011, Article ID 178568, 19 pages, 2011.

[4] E. A. Butcher, H. Ma, E. Bueler, V. Averina, and Z. Szabo, "Stability of linear time-periodic delay-differential equations via Chebyshev polynomials," International Journal for Numerical Methods in Engineering, vol. 59, no. 7, pp. 895-922, 2004.

[5] T. Diagana, "Existence of almost automorphic solutions to some neutral functional differential equations with infinite delay," Electronic Journal of Differential Equations, no. 129, p. 14, 2008.

[6] J. K. Hale and S. M. Verduyn Lunel, Introduction to FunctionalDifferential Equations, vol. 99 of Applied Mathematical Sciences, Springer, New York, NY, USA, 1993.

[7] T. Kalmár-Nagy, "Stability analysis of delay-differential equations by the method of steps and inverse Laplace transform," Differential Equations and Dynamical Systems, vol. 17, no. 1-2, pp. 185-200, 2009.

[8] F. Li and G. M. N’Guérékata, “An existence result for neutral delay integrodifferential equations with fractional order and nonlocal conditions," Abstract and Applied Analysis, vol. 2011, Article ID 952782, 20 pages, 2011.

[9] J. Liang, J. H. Liu, and T.-J. Xiao, "Periodic solutions of delay impulsive differential equations," Nonlinear Analysis. Theory, Methods \& Applications A, vol. 74, no. 17, pp. 6835-6842, 2011.

[10] J. Liang, H.-Y. Wang, and T.-J. Xiao, “On a comparison principle for delay coupled systems with nonlocal and nonlinear boundary conditions," Nonlinear Analysis. Theory, Methods \& Applications A, vol. 71, no. 12, pp. e359-e365, 2009.
[11] J. Liang and T.-J. Xiao, "Solutions to nonautonomous abstract functional equations with infinite delay," Taiwanese Journal of Mathematics, vol. 10, no. 1, pp. 163-172, 2006.

[12] J. Liang and T.-J. Xiao, "Solutions to abstract integral equations and infinite delay evolution equations," Bulletin of the Belgian Mathematical Society, vol. 18, no. 5, pp. 793-804, 2011.

[13] J. H. Liu, G. M. N'Guérékata, and N. V. Minh, Topics on Stability and Periodicity in Abstract Differential Equations, vol. 6 of Series on Concrete and Applicable Mathematics, World Scientific Publishing, Hackensack, NJ, USA, 2008.

[14] Y. Liu, W. Jiang, and F. Huang, "Robustness with respect to small delays for exponential stability of Pritchard-Salamon systems with admissible state feedback," International Journal of Evolution Equations, vol. 1, no. 3, pp. 225-263, 2005.

[15] P. H. A. Ngoc, N. van Minh, and T. Naito, "Stability radii of positive linear functional differential systems in Banach spaces," International Journal of Evolution Equations, vol. 2, no. 1, pp. 7597, 2007.

[16] P. Wahi and A. Chatterjee, "Galerkin projections for delay differential equations," Journal of Dynamic Systems, Measurement, and Control, vol. 127, pp. 80-89, 2005.

[17] T.-J. Xiao and J. Liang, "Blow-up and global existence of solutions to integral equations with infinite delay in Banach spaces," Nonlinear Analysis. Theory, Methods \& Applications A, vol. 71, no. 12, pp. e1442-e1447, 2009. 


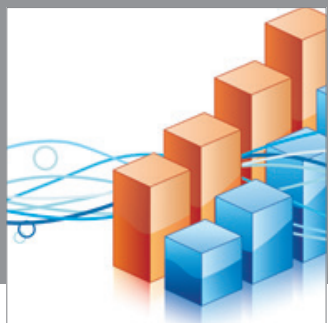

Advances in

Operations Research

mansans

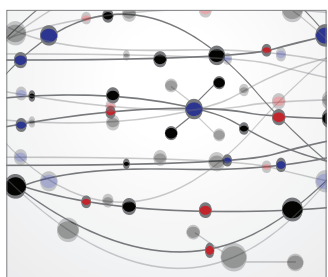

The Scientific World Journal
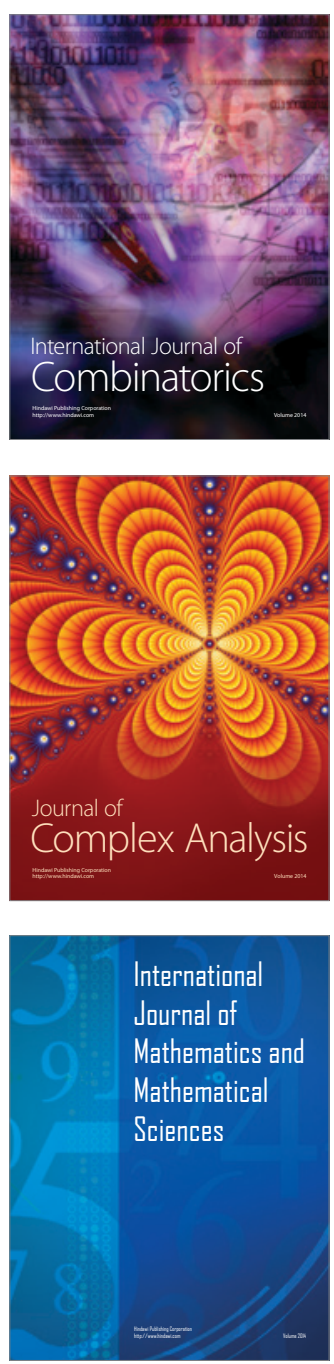
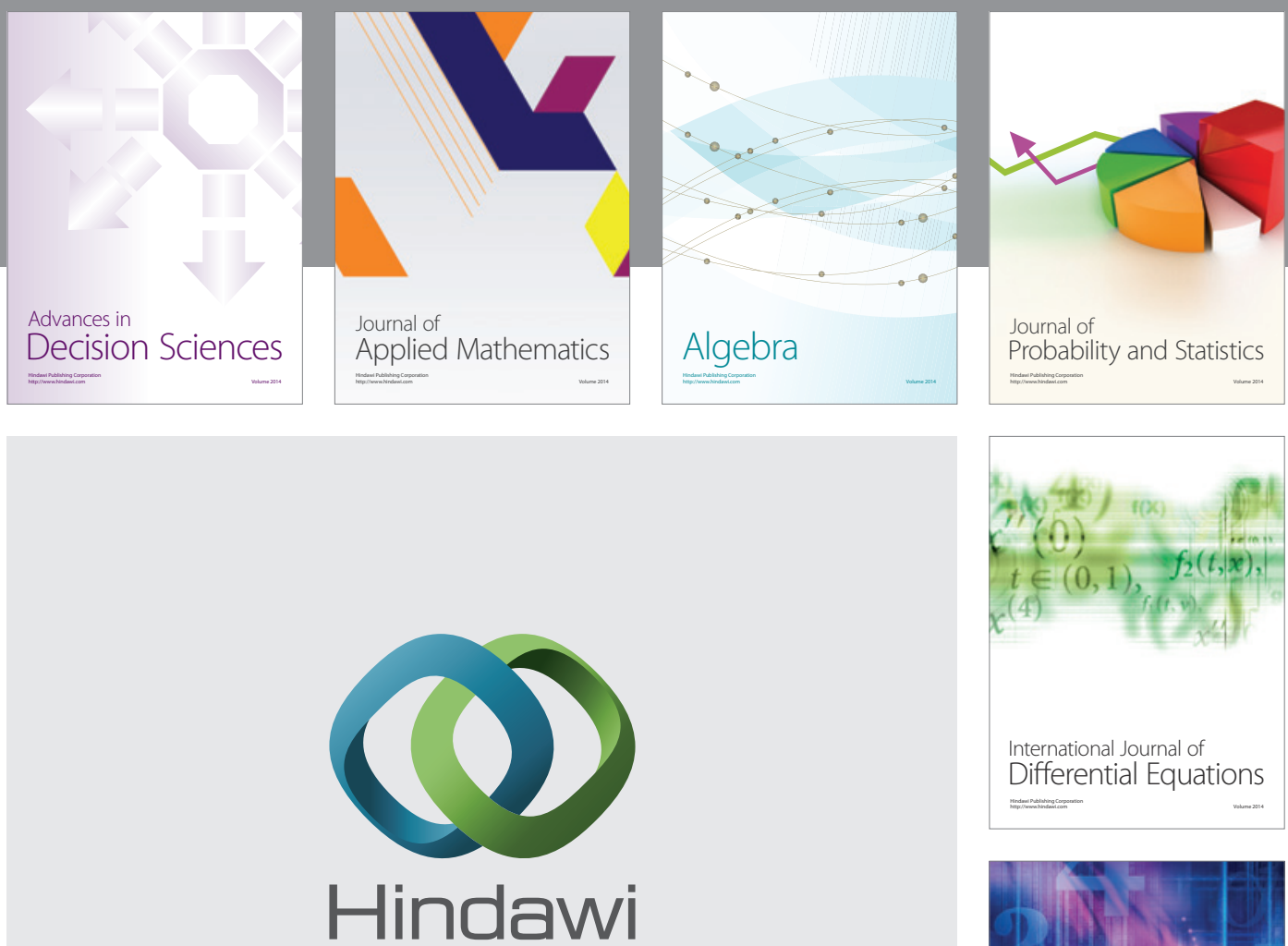

Submit your manuscripts at http://www.hindawi.com
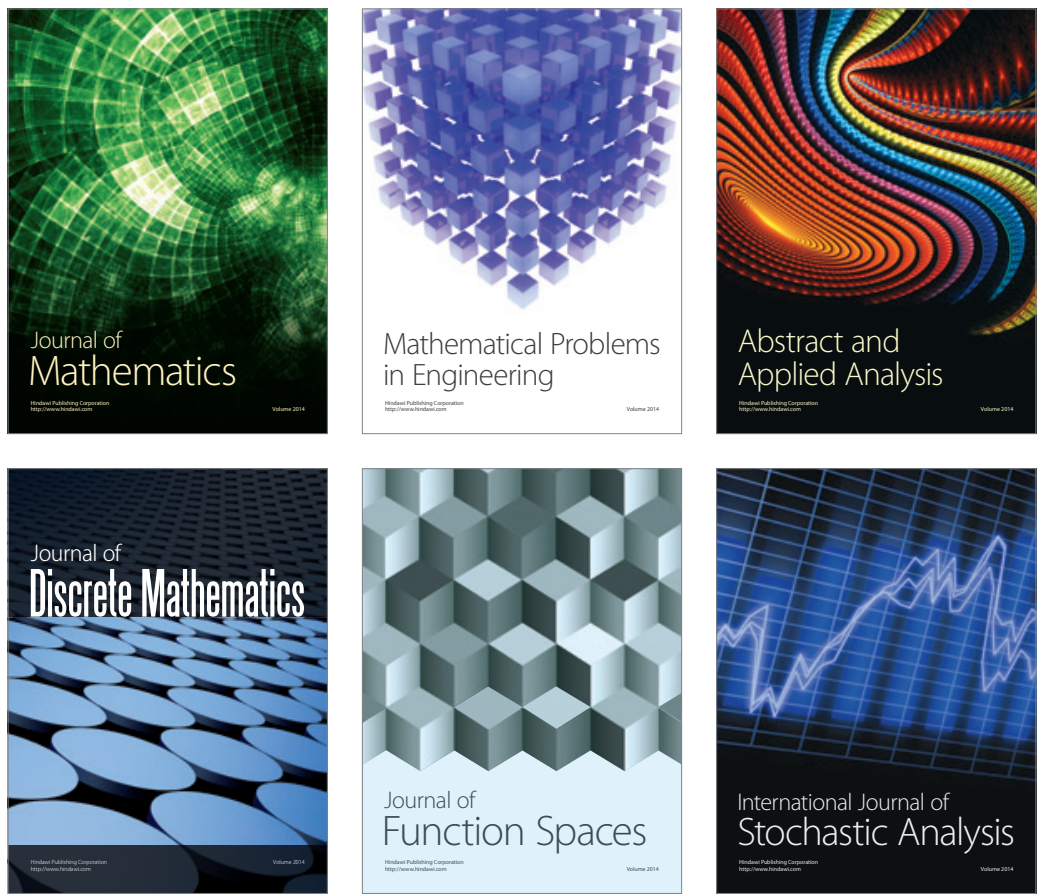

Journal of

Function Spaces

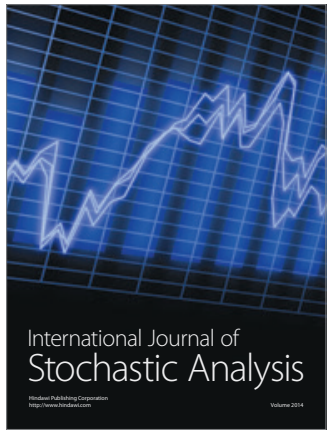

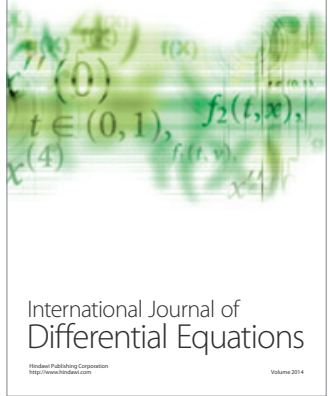
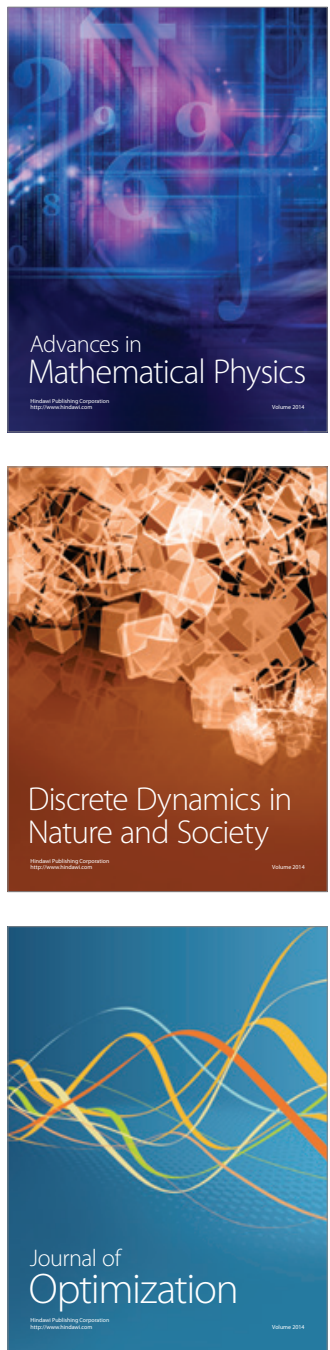